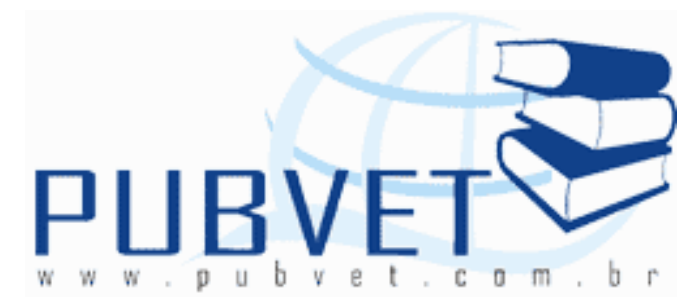

PUBVET, Publicações em Medicina Veterinária e Zootecnia.

\title{
Terminação de cordeiros
}

\section{José Carlos da Silveira Osório ${ }^{1,2,4}$; Maria Teresa Moreira Osório ${ }^{1,2}$; Sérgio} Silveira Gonzaga3; Carlos Eduardo da Silva Pedroso4; Roger Gomes Esteves ${ }^{2,4}$; Otoniel Geter Lauz Ferreira ${ }^{4}$; Fernando Miranda de Vargas Júnior ${ }^{5}$; Luis Gustavo Castro Alves ${ }^{5}$

1 Professor Visitante Nacional Sênior da Universidade Federal da Grande Dourados - CAPES. ${ }^{2}$ Bolsista CNPq. ${ }^{3}$ EMBRAPA, Pecuária Sul. ${ }^{4}$ Universidade Federal de Pelotas. ${ }^{5}$ Universidade Federal da Grande Dourados.

\section{Resumo}

O trabalho objetiva mostrar a importância e como determinar de maneira prática o momento em que o cordeiro está terminado a partir da relação entre a condição corporal do cordeiro e o estado de engorduramento da carcaça. Foram verificadas elevadas correlações entre estes fatores $(0,89)$, no entanto foram dependentes da experiência prévia do observador. Quando a técnica é efetuada por observadores menos experientes a correlação diminui de forma expressiva $(0,55)$, o que evidencia a necessidade de treinamento para execução desta técnica com sucesso. Deste modo, constata-se que a condição corporal é bom indicador do estado de engorduramento da carcaça e alternativa prática viável para determinar se o cordeiro está terminado.

Palavras-chave: condição corporal, estado de engorduramento, ovinos. 
OSÓRIO, J.C.S. et al. Terminação de cordeiros. PUBVET, Londrina, V. 6, N. 23, Ed. 210, Art. 1402, 2012.

\title{
Termination of lambs
}

\begin{abstract}
The objective work to show the importance and as to determine in practical way the moment where the lamb is finished from the relation enters the body condition of the lamb and the fattening status of the carcass. They had been verified raised correlations between these factors $(0,89)$, however had been dependents of the previous experience of the observer. When the technique is effected by less experienced observers the correlation diminishes of expressive form $(0,55)$, what it successfully evidences the necessity of training for execution of this technique. In this way, one evidences that the corporal condition is good pointer of the fattening status of the carcass and viable alternative practical to determine if the lamb is finished.
\end{abstract}

Keywords: body condition, carcass fattening state, sheep.

\section{INTRODUÇÃO}

Nos últimos anos na ovinocultura brasileira, a terminação de cordeiros passou a ter destaque em função dos bons preços praticados. Como consequência verifica-se intensa busca de tecnologia para otimização do processo produtivo e para colocar no mercado, cada vez mais exigente, carne de alta qualidade; aspecto imprescindível para competir com os demais produtos.

Para que se possa melhor entender a complexidade que envolve a terminação de cordeiro, é necessário que tenhamos, pelo menos, alguns aspectos bem claros; uma vez que "terminar o cordeiro" não é tão somente alimentar o animal. O processo envolve conhecer o animal, o alimento que the será ofertado nos diferentes períodos, determinar o momento propício para o abate e, também, o mercado e suas variações.

Pela complexidade que envolve o processo pergunta-se o que seria terminar cordeiro? 
OSÓRIO, J.C.S. et al. Terminação de cordeiros. PUBVET, Londrina, V. 6, N. 23, Ed. 210, Art. $1402,2012$.

Um cordeiro está terminado quando atende as necessidades do mercado. No do Cordeiro Herval Premium, hoje, o cordeiro está terminado quando apresenta condição corporal entre 2,5 e 3,5. Isto corresponde à carcaça com engorduramento entre 2,5 e 3,5, ou seja, animais e carcaças com quantidade e distribuição de gordura ligeiramente magra $(2,5)$ ou ligeiramente engordurada $(3,5)$, dentro da escala de 1 a 5 , como será apresentado mais adiante e já manifestado por Osório e Osório (2003).

A prática de atender as necessidades do consumidor já em nível de campo, a partir da condição corporal dos animais, está sendo utilizada com sucesso nos últimos cinco anos pelo Conselho Regulador do Cordeiro Herval Premium. Resultados publicados por Osório et al.(2004) comprovam a eficiência entre a avaliação in vivo e da carcaça e o consequente atendimento ao que o mercado solicita.

Portanto, quando o foco é o consumidor, "terminar cordeiro" é diferente de "engordar cordeiro"; embora sejam aceitas as considerações e distintos pontos de vista que colocam esses termos como sinônimos. Nesse sentido, levamos em consideração que o cordeiro pode estar gordo e não estar terminado ou pode-se engordar um cordeiro terminado; e, certamente, abater cordeiro gordo ou engordar cordeiro terminado é um custo desnecessário e oneroso para o processo produtivo.

Outro aspecto importante: como determinar se o cordeiro está terminado?

Pode ser através da condição corporal do cordeiro e pelo correspondente estado de engorduramento da carcaça deste.

A condição corporal foi definida por Murray (1919) como a quantidade de gordura e dos demais tecidos no organismo do animal vivo. Atualmente, em animais destinados ao abate a condição corporal busca estimar a relação músculo/gordura, fornecendo um entendimento entre o vendedor de carne e aquele que determina o momento de abate do animal. Portanto, busca-se na condição corporal uma avaliação do estado de engorduramento da carcaça. 
OSÓRIO, J.C.S. et al. Terminação de cordeiros. PUBVET, Londrina, V. 6, N. 23, Ed. 210, Art. $1402,2012$.

Para apreciação da condição corporal é utilizada a palpação de regiões corporais do animal (Figura 1 e 2), que refletem o estado dos diferentes depósitos de gorduras. A comissão britânica de produção de carne (Meat and Livestock Commission - MLC, 1983) elaborou uma metodologia científica subjetiva para estimar o estado de engorduramento no cordeiro vivo. Os pontos de palpação são (Cañeque et al., 1989): A - tronco da cola, por ser a última parte a depositar gordura, reflete o estado de engorduramento corporal. Quando se palpa essa região, busca-se detectar os ossos; maior dificuldade em senti-los, significa um maior engorduramento. B - ao longo das apófises espinhosas lombares e sobre o músculo Longissimus dorsi e as pontas das apófises transversas lombares. Colocando-se a mão sobre elas, busca-se sentir sua proeminência; quanto menos, maior é o engorduramento. C - ao longo das apófises espinhosas dorsais. D - ao longo do esterno.

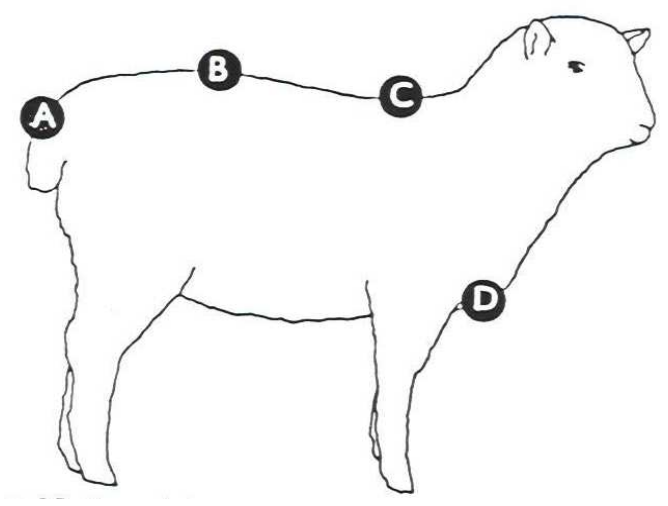

Figura 1. Pontos de palpação para estimar a condição corporal no cordeiro.

$\mathrm{Na}$ estimava da condição corporal é atribuído índice de 1 a 5, com subdivisões de 0,5 em 0,5 (Tabela 1), similar à escala dos índices do estado de engorduramento da carcaça. Mas, cabe ressaltar, que o índice ideal será aquele que corresponda á preferência do mercado consumidor. 
OSÓRIO, J.C.S. et al. Terminação de cordeiros. PUBVET, Londrina, V. 6, N. 23, Ed. 210, Art. 1402, 2012.

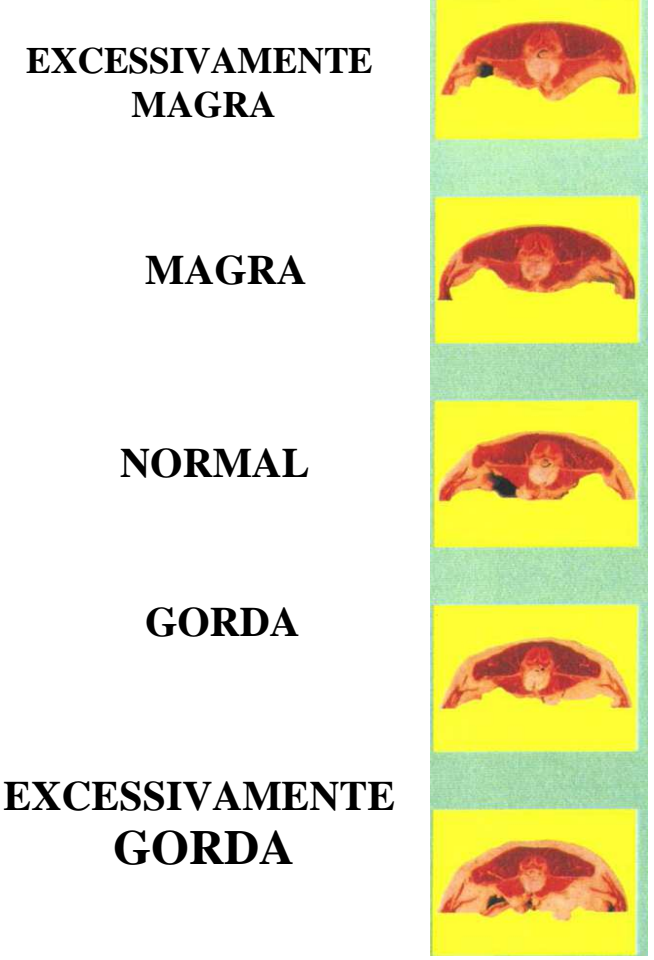

Figura 2. Condição corporal segundo as apófises espinhosas e lombares transversas.

Tabela 1. Descrição da escala de condição corporal.

\begin{tabular}{|c|c|}
\hline ÍNDICE & DESCRIÇÃO \\
\hline 1,0 & EXCESSIVAMENTE MAGRA \\
\hline 1,5 & MUITO MAGRA \\
\hline 2,0 & MAGRA \\
\hline 2,5 & LIGEIRAMENTE MAGRA \\
\hline 3,0 & NORMAL \\
\hline 3,5 & GIGEIRAMENTE ENGORDURADA \\
\hline 4,0 & MUITO GORDA \\
\hline 4,5 & EXCESSIVAMENTE GORDA \\
\hline 5,0 & \\
\hline
\end{tabular}

Osório et al., 1998; Osório e Osório, 2003. 
OSÓRIO, J.C.S. et al. Terminação de cordeiros. PUBVET, Londrina, V. 6, N. 23, Ed. 210, Art. 1402, 2012.

O estado de engorduramento da carcaça é feito por apreciação visual, avaliando-se a gordura de cobertura em quantidade e distribuição.

$\mathrm{Na}$ avaliação do estado de engorduramento da carcaça atribui-se um índice de 1 a 5, com subdivisão de 0,5 em 0,5 (Tabela 2). Na prática, sempre que possível, deve-se fazer uma avaliação comparativa com ordenação das carcaças ou com padrões fotográficos.

Tabela 2. Descrição da escala do estado de engorduramento da carcaça.

\begin{tabular}{|c|c|}
\hline ÍNDICE & DESCRIÇÃO \\
\hline 1,0 & EXCESSIVAMENTE MAGRA \\
\hline 1,5 & MUITO MAGRA \\
\hline 2,0 & MAGRA \\
\hline 2,5 & LIGEIRAMENTE MAGRA \\
\hline 3,0 & NORMAL \\
\hline 3,5 & GIGEIRAMENTE ENGORDURADA \\
\hline 4,0 & MUITO GORDA \\
\hline 4,5 & EXCESSIVAMENTE GORDA \\
\hline 5,0 &
\end{tabular}

De maneira que, o estado de engorduramento da carcaça pode ser usado como elo para o entendimento entre o que o consumidor deseja de gordura e o que o produtor pode conseguir, através da condição corporal do animal.

O presente estudo objetivou mostrar a importância da condição corporal como indicativo da terminação do cordeiro e sua relação com o estado de engorduramento da carcaça.

\section{MATERIAL E MÉTODOS}

Foram utilizados dados de dois abates de 136 ovinos que não romperam as pinças permanentes (idade inferior a um ano), considerados "cordeiros", comercializados com a Denominação de Carne Ovina de Qualidade "Cordeiro Herval Premium". Os abates foram no Matadouro Frigorífico BonSul, Pelotas, 
OSÓRIO, J.C.S. et al. Terminação de cordeiros. PUBVET, Londrina, V. 6, N. 23, Ed. 210, Art. $1402,2012$.

Rio Grande do Sul, nos dias 15 e 29 de março de 2004 com, 66 e 70 cordeiros abatidos, respectivamente.

Antes de serem transportados (caminhão) para o frigorífico, os cordeiros foram avaliados quanto à condição corporal por técnicos do Conselho Regulador do Cordeiro Herval Premium, cujo objetivo foi selecionar animais de acordo com a exigência de mercado, ou seja, animais com índices entre 2,5 e 3,5 (amplitude de 1 a 5).

No dia do abate, os cordeiros foram identificados e pesados individualmente e, a seguir avaliados "in vivo", por palpação, a condição corporal (índice de 1 a 5 ) por técnicos treinados.

Foi avaliada a conformação visual do cordeiro, por um dos técnicos, atribuindo índice de 1 a 5 , com subdivisões de 0,5 em 0,5 , onde 1 é muito pobre e 5 é excelente.

Foi tomado o comprimento corporal dos cordeiros, em centímetros. A partir deste, foi determinada á compacidade corporal (peso corporal dividido pelo comprimento corporal, em $\mathrm{kg} / \mathrm{cm}$ ).

Após, os cordeiros foram abatidos e, imediatamente, tomado o peso de carcaça quente; sendo que, a partir do peso corporal e da carcaça foi calculado o rendimento de carcaça no frigorífico (peso de carcaça quente dividido pelo peso corporal vezes 100).

A seguir, foi avaliado o estado de engorduramento da carcaça pelo técnico do Conselho Regulador, atribuindo índice de 1 a 5, com subdivisões de 0,5 em 0,5 , onde 1 é excessivamente magra e 5 é excessivamente gorda. Esta e as demais avaliações estão descritas por Osório e Osório (2003, 2005).

Através do Proc CORR do programa SAS (2001) foram estimados os coeficientes de correlações entre o estado de engorduramento, condição corporal e demais características. Também foram obtidas distribuições de freqüências da condição corporal e do estado de engorduramento.

O delineamento experimental utilizado foi o inteiramente ao acaso. 
OSÓRIO, J.C.S. et al. Terminação de cordeiros. PUBVET, Londrina, V. 6, N. 23, Ed. 210, Art. $1402,2012$.

\section{RESULTADOS}

No abate do dia 15.03.2004, foram avaliados 66 cordeiros quanto a condiçãocorporal por dois técnicos ( $\mathrm{CC} 1$ e CC2) e estado de engorduramento pelo técnico do Conselho Regulador (EE).

Na Tabela 3 apresenta-se a distribuição do número e percentagem de cordeiros em cada índice de condição corporal e estado de engorduramento da carcaça, atribuído por cada técnico.

Tabela 3. Distribuição numérica e percentual da condição corporal e estado de engorduramento da carcaça (EE).

\begin{tabular}{|l|c|c|c|}
\hline Índice & \multicolumn{2}{|c|}{ Condição corporal } & \multirow{2}{*}{ EE } \\
\hline 1,0 & Avaliador CC1 & Avaliador CC2 & $1=1,54 \%$ \\
\hline 1,5 & 0 & 0 & $2=3,08 \%$ \\
\hline 2,0 magra & $21=31,82 \%$ & $15=22,73 \%$ & $6=9,23 \%$ \\
\hline 2,5 & $28=42,42 \%$ & $26=39,39 \%$ & $18=27,69 \%$ \\
\hline 3,0 normal & $12=18,18 \%$ & $19=28,79 \%$ & $36=55,38 \%$ \\
\hline 3,5 & $2=3,03 \%$ & $4=6,06 \%$ & $2=3,08 \%$ \\
\hline Total & $66=100 \%$ & $66=100 \%$ & $65=100 \%$ \\
\hline
\end{tabular}

Observa-se que os percentuais de cordeiros com condição corporal e estado de engorduramento da carcaça excessivamente magros (índice 1) e muito magros (índice 1,5) foi inferior a 4,62\%. Espera-se terminar, em função da preferência de mercado, com o sacrifício de cordeiros com esses índices; uma vez que, além de não ser a preferência do consumidor, com ganho de peso de $150 \mathrm{~g} /$ dia, o produtor em mais dez dias poderia ganhar $1,5 \mathrm{~kg}$ e propiciar a satisfação do consumidor em sua plenitude. Entre os índices 2 (magra) e 3 (normal), há necessidade de estudo mais detalhado. Porém, certamente, a utilização de pastagem melhorada resultará na otimização do sistema e, por consequência, no aumento percentual de animais com índices 3 e 3,5 . 
OSÓRIO, J.C.S. et al. Terminação de cordeiros. PUBVET, Londrina, V. 6, N. 23, Ed. 210, Art. $1402,2012$.

Também, parece importante ressaltar que: a) acima de $90 \%(92,42 \%$ do CC1, $90,91 \%$ do CC2 e $92,30 \%$ do EE) dos animais e de suas carcaças, estão entre os índices 2 e 3. b) o percentual de carcaças magras, índice 2, embora expressivo $(9,23 \%)$ é bem inferior ao mesmo índice atribuído na condição corporal e, isso necessita ser ajustado. c) o percentual avaliado como índice 3 é superior nas carcaças $(55,38 \%)$ em relação ao do animal $(18,18 \%$ e 28,79\%, respectivamente para os avaliadores da condição corporal, CC1 e CC2).

Na Tabela 4, verifica-se que 89,23\% (CC1) e 93,82\% (CC2) da avaliação "in vivo" é igual ou mais ou menos 0,5 da avaliação da carcaça.

Tabela 4. Distribuição numérica e percentual da condição corporal (CC1 e CC2) e estado de engorduramento da carcaça (EE).

\begin{tabular}{|l|l|l|}
\hline EE & Avaliador CC1 & Avaliador CC2 \\
\hline Igual & $20=30,77 \%$ & $26=40,00 \%$ \\
\hline Mais 0,5 & $32=49,23 \%$ & $24=36,92 \%$ \\
\hline Menos 0,5 & $6=9,23 \%$ & $11=16,92 \%$ \\
\hline Mais 1 & $6=9,23 \%$ & $3=4,62 \%$ \\
\hline Menos 1 & $1=1,54 \%$ & $1=1,54 \%$ \\
\hline
\end{tabular}

Igualmente, corroborando com os percentuais apresentados (Tabela 4), que indicam relação excelente entre a avaliação "in vivo" e da carcaça, estão os coeficientes de correlação entre essas características (Tabela 5). Sendo que, os coeficientes de correlação entre a condição corporal do animal e o estado de engorduramento da carcaça são superiores aos obtidos entre o peso corporal do animal e o estado de engorduramento da carcaça. 
OSÓRIO, J.C.S. et al. Terminação de cordeiros. PUBVET, Londrina, V. 6, N. 23, Ed. 210, Art. 1402, 2012.

Tabela 5. Coeficientes de correlação, da condição corporal avaliada por dois técnicos (CC1 e CC2) e estado de engorduramento da carcaça (EE).

\begin{tabular}{|l|l|l|l|}
\hline & CC 1 & CC 2 & EE \\
\hline CC 2 & $0,84^{* *}$ & & \\
\hline EE & $0,55^{* *}$ & $0,57 * *$ & \\
\hline Peso corporal & $0,45^{* *}$ & $0,43^{* *}$ & $0,45^{* *}$ \\
\hline Comprimento corporal & $0,31^{* *}$ & $0,04 \mathrm{~ns}$ & $0,38^{* *}$ \\
\hline Peso de carcaça quente & $0,36^{* *}$ & $0,43^{* *}$ & $0,32^{* *}$ \\
\hline Rendimento de carcaça & $-0,07^{\mathrm{ns}}$ & $0,06 \mathrm{~ns}$ & $-0,13^{\mathrm{ns}}$ \\
\hline Compacidade corporal & $0,28^{*}$ & $0,40^{* *}$ & $0,25^{*}$ \\
\hline
\end{tabular}

$* *=(\mathrm{P}<0,01) . *=(\mathrm{P}<0,05) . \mathrm{ns}=(\mathrm{P}>0,05)$.

Entre os avaliadores da condição corporal (CC1 e CC2) o coeficiente de correlação é indicativo do entendimento na avaliação e foi superior aos obtidos nos estudos de Bonacina et al. (2007) e Esteves et al. (2010), que foram de 0,50 no de Bonacina e de $0,35,0,41,0,45,0,48,0,53$ e 0,54 no de Esteves, já que neste a condição corporal foi por quatro avaliadores. Entretanto, o coeficiente de correlação do presente estudo (Tabela 5) é similar aos obtidos por Hashimoto et al. (2007), que entre quatro avaliadores da condição corporal foram: $0,77,0,83,0,83,0,84,0,86$ e 0,90 . Mas, os coeficientes de correlação deste estudo, do segundo abate (Tabela 8 ) são maiores, entre três avaliadores da condição corporal foram de 0,90, 0,92 e 0,95 (os avaliadores da condição corporal do segundo abate participam desde a criação do Conselho Regulador do Cordeiro Herval Premium, veterinários e agrônomo, com larga experiência).

Pelo exposto e com base nos resultados, que indicaram a importância do treinamento dos avaliadores, pode 90,25\% (coeficiente de determinação, $R^{2}$, do coeficiente de correlação, $r=0,95)$ da variação do estado de engorduramento da carcaça ser devido à variação da condição corporal do animal.

Importante salientar que os animais já haviam sido avaliados na propriedade rural, onde foram eliminados os extremos e selecionados os aptos 
OSÓRIO, J.C.S. et al. Terminação de cordeiros. PUBVET, Londrina, V. 6, N. 23, Ed. 210, Art. $1402,2012$.

para comercialização dentro da marca de qualidade. Tal evento diminui os valores dos coeficientes de correlação, já que os extremos seriam facilmente detectados.

No abate do dia 29.03.2004, avaliados 70 cordeiros foram avaliados por três técnicos (CC1, CC3 e CC4) de campo, quanto à condição corporal e, o estado de engorduramento, por um quarto técnico (EE).

Diferente do abate de 15/03/04, nenhum cordeiro foi classificado com o índice 1 (Tabela 6); mas, a de carcaças com índice 1,5 (muito magra) aumentou de $4,62 \%$ para $7,14 \%$. Por outro lado, a $\%$ de carcaças com índice 3,5 (ligeiramente engordurada) também aumentou $e$, inclusive, foram verificados animais com índices acima de 4 (34,23\% das carcaças).

Tabela 6. Distribuição numérica e percentual da condição corporal para cada avaliador (CC1, CC3 e CC4) e estado de engorduramento da carcaça (EE).

\begin{tabular}{|c|c|c|c|c|}
\hline Índice & CC1 & $\mathrm{CC} 3$ & CC4 & EE \\
\hline 1,5 & $4=5,71 \%$ & & $1=1,43 \%$ & $5=7,14 \%$ \\
\hline 2,0 & $12=17,14 \%$ & $14=20,00 \%$ & $14=20 \%$ & $10=14,29 \%$ \\
\hline 2,5 & $16=22,86 \%$ & $15=21,43 \%$ & $15=21,43 \%$ & $9=12,86 \%$ \\
\hline 3,0 & $11=15,71 \%$ & $7=10,00 \%$ & $6=8,57 \%$ & $14=20 \%$ \\
\hline 3,5 & $14=20,00 \%$ & $11=15,71 \%$ & $12=17,14 \%$ & $8=11,43 \%$ \\
\hline 4,0 & $12=17,14 \%$ & $18=25,71 \%$ & $12=17,14 \%$ & $5=7,14 \%$ \\
\hline 4,5 & $1=1,43 \%$ & $5=7,14 \%$ & $9=12,866 \%$ & $9=12,86 \%$ \\
\hline 5,0 & & & $1=1,43 \%$ & $10=14,29 \%$ \\
\hline Total & $70=100 \%$ & $70=100 \%$ & $70=100 \%$ & $70=100 \%$ \\
\hline
\end{tabular}

Isso ocorreu em função de um lote da raça Texel, com 32 cordeiros, apresentar média de 4,3 de estado de engorduramento com amplitude de 3,5 a 5, totalmente atípico, que deveriam ter sido abatidos antes. Carcaças essas, com problemas de colocação no mercado consumidor atendido pela distribuidora Timers, fora do padrão do Cordeiro Herval Premium. 
OSÓRIO, J.C.S. et al. Terminação de cordeiros. PUBVET, Londrina, V. 6, N. 23, Ed. 210, Art. $1402,2012$.

Se animais da raça Texel, com menos de 12 meses de idade, podem alcançar índices de engorduramento da carcaça acima de 3,5, certamente, pode-se pensar que, caso o mercado tenha preferência por maiores quantidades de gordura nas carcaças, atingir este mercado É POSSÍVEL.

Apesar do problema anteriormente citado, na avaliação "in vivo" de três técnicos $(55,71 \%, 51,43 \%$ e $50 \%$, respectivamente), os índices da condição corporal foram entre 2 e 3 e 47,15\% das carcaças apresentaram estado de engorduramento entre os índices 2 e 3.

Quanto à relação entre os avaliadores, Tabela 7, verifica-se que 65,72\% (CC1), 83,14\% (CC3) e 88,58\% (CC4) da avaliação "in vivo" é igual ou mais ou menos 0,5 da avaliação da carcaça. Valores pouco inferiores aos verificados no primeiro abate (Tabela 4), todavia ótimos quanto à correspondência entre os técnicos.

Tabela 7. Número e percentual de índices atribuídos na avaliação da condição corporal (CC1, CC3 e CC4) conforme o estado de engorduramento da carcaça (EE).

\begin{tabular}{|l|l|l|l|}
\hline EE & CC1 & CC3 & CC4 \\
\hline Igual & $12=17,14 \%$ & $21=30,00 \%$ & $21=30,00 \%$ \\
\hline Mais 0,5 & $24=34,29 \%$ & $25=31,71 \%$ & $24=34,29 \%$ \\
\hline Menos 0,5 & $10=14,29 \%$ & $15=21,43 \%$ & $17=24,29 \%$ \\
\hline Mais 1 & $19=27,14 \%$ & $5=7,14 \%$ & $6=8,57 \%$ \\
\hline Menos 1 & $3=4,29 \%$ & $2=2,86 \%$ & $1=1,43 \%$ \\
\hline Mais 1,5 & $2=2,86 \%$ & $2=2,86 \%$ & $1=1,43 \%$ \\
\hline
\end{tabular}

Cabe salientar que existem fatores que podem influir sobre a avaliação da condição corporal, ou seja, dificultando um pouco essa avaliação, como, por exemplo, a quantidade de lã (animais com muita lã, lã inteira, em relação aos sem lã), familiaridade com a raça ou raças distintas em um mesmo momento, entre outros. 
OSÓRIO, J.C.S. et al. Terminação de cordeiros. PUBVET, Londrina, V. 6, N. 23, Ed. 210, Art. $1402,2012$.

Quanto aos coeficientes de correlação, Tabela 8, entre os avaliadores (CC1, CC3, CC4 e EE) os resultados são superiores aos do abate anterior e isso ocorreu não somente em função de ajustes; mas, também, por apresentar um lote (Texel), salientado anteriormente, marcadamente diferenciado, ou seja, os extremos são bem percebidos e há uma melhora da avaliação e, o contrário, também é verdadeiro, ou seja, pequenas diferenças, animais similares, são mais difíceis de diferenciar com precisão.

Tabela 8. Coeficientes de correlação, da condição corporal avaliada por três técnicos (CC1, CC3 e CC4) e estado de engorduramento da carcaça (EE).

\begin{tabular}{|l|l|l|l|l|}
\hline & CC1 & CC3 & CC4 & EE \\
\hline CC 3 & $0,90 * *$ & & & \\
\hline CC 4 & $0,92 * *$ & $0,95 * *$ & & \\
\hline EE & $0,85 * *$ & $0,89 * *$ & $0,89 * *$ & \\
\hline Conformação in vivo & $0,88 * *$ & $0,88 * *$ & $0,89 * *$ & $0,89 * *$ \\
\hline Peso corporal & $0,71 * *$ & $0,76 * *$ & $0,75 * *$ & $0,83 * *$ \\
\hline Comprimento corporal & $0,53 * *$ & $0,63 * *$ & $0,62 * *$ & $0,75 * *$ \\
\hline Peso de carcaça quente & $0,82 * *$ & $0,85 * *$ & $0,85 * *$ & $0,90 * *$ \\
\hline Rendimento de carcaça & $0,74 * *$ & $0,73 * *$ & $0,74 * *$ & $0,69 * *$ \\
\hline Compacidade corporal & $0,70 * *$ & $0,72 * *$ & $0,70 * *$ & $0,75 * *$ \\
\hline
\end{tabular}
$* *=(\mathrm{P}<0,01)$.

Os coeficientes de correlação entre o peso e condição corporal mostram que até $57,96 \%$ da variação do peso pode ser devido a variação da condição corporal; bem como $80,25 \%$ da variação do peso de carcaça pode ser devido a variação da condição corporal. Também se observa que com o aumento da condição corporal há maior comprimento e rendimento de carcaça bem como aumento da compacidade corporal. Portanto, a seleção, escolha e/ou fixação de pesos corporais, de carcaça, rendimento ou compacidade e conformação de cordeiros influi na condição corporal e, consequentemente no estado de engorduramento da carcaça. Logo, desconsiderar essas implicações biológicas 
OSÓRIO, J.C.S. et al. Terminação de cordeiros. PUBVET, Londrina, V. 6, N. 23, Ed. 210, Art. $1402,2012$.

pode levar a obtenção de carne com baixa qualidade, considerando que o estado de engorduramento é determinante da preferência do consumidor e a gordura é responsável pelo sabor (Mottram, 1998; Madruga et al., 2001).

Mas, não basta somente saber determinar se o animal está terminado, deve-se saber, também, quando o animal responde mais eficientemente a uma alimentação. Assim, é importante conhecimentos sobre o animal, alimento e mercado.

Quanto ao animal, é sabido que ao aproximar-se da maturidade os ganhos de peso diminuem e que a deposição de músculo ocorre antes da gordura e a deposição de gordura é mais cara do que a de músculo. Estudos indicam que para deposição de $1 \mathrm{~kg}$ de tecido adiposo é necessário de 10 a 20 vezes mais quilos de calorias do que para depositar $1 \mathrm{~kg}$ de tecido muscular.

Portanto, de acordo com observações e estudos, os animais devem ser abatidos ou terminados a $40-60 \%$ do seu peso corporal adulto.

Sendo que para essa terminação utiliza-se uma gama de alimentos, desde pastagem nativa melhorada, pastagens cultivadas e subprodutos agrícolas e industriais até o fornecimento de alimentos concentrados.

As situações são as mais variadas e o sistema a ser adotado deve levar em consideração as disponibilidades dos insumos. Como exemplo cita-se a utilização do azevém anual como fonte de alimento em períodos de entressafras de arroz ou soja.

Outra alternativa é a integração da ovinocultura com a silvicultura e fruticultura, pois geram interações fundamentais para a redução dos custos operacionais ambientais e para a viabilização econômica do sistema de produção.

\section{CONCLUSÕES}

Para obtenção de carne de qualidade deve-se terminar o cordeiro pensando no consumidor e na oferta constante de produto uniforme e de qualidade. 
OSÓRIO, J.C.S. et al. Terminação de cordeiros. PUBVET, Londrina, V. 6, N. 23, Ed. 210, Art. $1402,2012$.

A condição corporal do animal é indicador eficaz para estimar o estado de engorduramento do cordeiro.

\section{BIBLIOGRAFIA}

Bonacina, M.; Osório, J.C.S.; Osório, M.T.M. et al. Otimização da avaliação in vivo e da carcaça em cordeiros. Revista da Faculdade Zootecnia, Veterinária e Agronomia, Uruguaiana, v.14, n.1, p.273-286, 2007.

Cañeque, V.; Huildobro, F.R.; Dolz, J.F. et al. Producción de Carne de Cordero. Ministério de Agricultura Pesca y Alimentación. Colección Técnica. España. 520 p. 1989.

Esteves, R.M.; Osório, J.C.S.; Osório, M.T.M. et al. Avaliação in vivo e da carcaça e fatores determinantes para o entendimento da cadeia da carne ovina. Revista Brasileira de Agrociência, v.16, n.1-4, p.101-108, 2010.

Hashimoto, J.H.; Osório, J.C.S.; Osório, M.T.M. et al. Avaliação in vivo e da carcaça de cordeiros Corriedale de diferentes sexos. PUBVET, Londrina, v.4, n.1, Ed. 106, Art. 713, 2010. Disponível em: http://www.pubvet.com.br/artigos det.asp?artigo=591.

Madruga, M.S.; Narain, N.; Souza, J.G. et al. Castration and slaughter age effects on fat components of "Mestiço" goat meat. Small Ruminant Research, v.42, n.1, p.75-80, 2001.

Meat and Livestock Commission. Lamb Carcase Production. Sheep Carcase Classification. 1983.

Mottram, D.S. Flavour formation in meat and meat products: a review. Food Chemistry, v.62, n.4, p.415-424, 1998.

Murray, J.A. Meat production. Journal of Agricultural Science, Cambridge, v.9, p.174-181, 1919.

Osório, J.C.S.; Osório, M.T.M.; Jardim, P.O.C. et al. Métodos para avaliação da produção de carne ovina: in vivo, na carcaça e na carne. Editora e Gráfica Universitária - UFPEL. Pelotas, RS. 1998. 107 páginas.

Osório, J.C.S.; Osório, M.T.M.; Oliveira, N.M. et al. Qualidade, Morfologia e Avaliação de Carcaças. Editora e Gráfica Universitária - UFPEL. Pelotas, RS. 2002, 195 páginas.

Osório, J.C.S.; Osório, M.T.M. Produção de Carne Ovina: Técnicas de Avaliação "in vivo" e na Carcaça. Editado por José Carlos da Silveira Osório. 2003. 73 páginas.

Osório, J.C.S.; Correa, F.; Osório, M.T.M. et al. Avaliação in vivo e da carcaça em cordeiro. XXXIO Congresso Brasileiro de Medicina Veterinária, São Luís - MA, Anais...CD-ROM. 2004.

Osório, J.C.S.; Osório, M.T.M. Produção de carne ovina: Técnicas de avaliação "in vivo" e na carcaça. 2a ed. Pelotas: Universidade Federal de Pelotas. Ed. Universitária, 82p. 2005.

SAS INSTITUTE INC. System for Microsoft Windows. Release 8.01. Cary: NC, USA, $2001-$ CD-ROM. 